\title{
Selection of coordinate axis and calculation of coordinate point direction in apparel CAD system
}

\author{
Xiaolu Ye \\ College of Design and Creativity, Wenzhou Polytechnic, Wenzhou \\ 325003,Zhejiang, China
}

\begin{abstract}
In the clothing CAD system, the grading of the clothing pattern occupies a very important position. In this paper,we enumerated and analyzed the problems that often occur in the operation of pattern grading CAD system. And focused on how to select the vertical and horizontal coordinate axes and calculate the plus and minus signs of the grading points of clothing pattern before grading and inputting the grading values in pattern grading CAD system, and then brought them into the women's suit pattern for application and verification. At last achieved the purpose of improving the efficiency of pattern grading.
\end{abstract}

KEYWORDS: pattern grading CAD system, grading technology, coordinate axis, calculation of the sign

\section{Introduction}

With the development of social mass production in today's garment industry, it is required that the same style of clothing should be organized and produced according to a variety of specifications or size series to adapt to different wearing objects and meet the needs of most consumers. On this basis, the technology of costume pattern grading came into being. In foreign countries, the development of costume pattern grading technology began with the first computer-aided garment design software developed in the United States in the early 1970s [1]. It is an important parameter to measure the CAD system. The effect and operation of pattern grading is also one of the issues that CAD users are very concerned about [2] Since 1980s, the function module of pattern grading has been widely used in garment enterprises all over the world, which has greatly improved the production

Published by Francis Academic Press, UK 
Academic Journal of Computing \& Information Science

ISSN 2616-5775 Vol. 3, Issue 1: 102-113, DOI: 10.25236/AJCIS.2020.030111

efficiency of garment enterprises. At present, it has been basically popularized in developed countries. In China, in the 1990s, the pattern grading CAD system with independent intellectual property rights was established in the National Tenth Five-Year Plan. After 2000, the domestic pattern grading CAD technology developed rapidly [3]. At present, pattern grading CAD technology is developing towards the direction of intelligent, three-dimensional and rapid response.

The pattern grading function in the CAD system can be divided into size division method, point grading method, cut line grading method and so on according to different operation methods [4]. One of the most commonly used and understandable methods is the point grading method. The process of point grading is to first determine the key points for grading on the basic template. These key points are sufficient and necessary points that can uniquely determine the shape and size of the pattern. Then calculate the grading value of each key point according to the specifications and grades, and enter CAD for grading [5]. However, in the use of the point grading system, it is found that when converting to the pattern grading CAD system for grading value input, the size pattern is often confused, or even completely wrong, even though the initial grading point values are calculated correctly. This phenomenon has brought great difficulty to the grading process of garment industry and reduced the efficiency.This paper will try to analyze and solve the problems that we found.

\section{Discussion on problems}

The pattern grading value includes the pattern grading quantity and direction. The pattern grading value and direction of the pattern grading point in the pattern will be different if the datum line and datum point selected in the basic pattern are different [6]. Therefore, before grading, first select the datum line to establish the two-dimensional rectangular coordinate system according to the convenience of grading.Then, the pattern grading value of each pattern grading point is calculated according to the rules, algorithms and grade in national standards or enterprises. Then, according to the position of the pattern grading point in the coordinate system, we can get the direction sign of the pattern grading value [7]. In the pattern grading CAD system, the value and sign of the grading point need to be input at the same time. If the operator can not judge the positive and negative sign of the pattern

Published by Francis Academic Press, UK 
Academic Journal of Computing \& Information Science

ISSN 2616-5775 Vol. 3, Issue 1: 102-113, DOI: 10.25236/AJCIS.2020.030111

grading point clearly, the size of the same set of pattern or within the same pattern will be confused. Here are a few typical examples.

If the pattern be graded correctly, the straight lines, curves and arcs of the the series pattern in the same parts should be kept parallel. However, in Figure 1, the two ends of the slacks bottom of back trousers are not straight because of the opposite size cause the line crossing. The grade direction is the wrong on the same line of the same pattern.

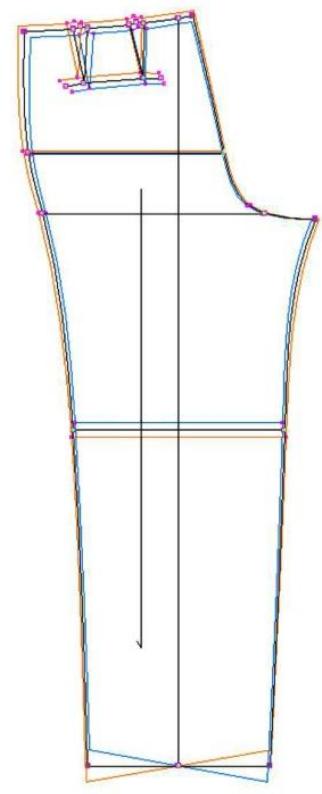

Figure. 1 The pattern of back trousers

Figure 2 is the front of men's shirt. At the beginning of the pattern setting, the red line indicates small size, and the blue line indicates large size. After pattern grading according to the datum line selected by the pattern, the correct situation is that the outline of the large size is outside the datum size, on the contrary, the small size is inside the datum line. However, the small size of the shoulder line, armhole arc line and side seam line of the pattern in Figure 2 is outside the datum size, while the large size is within the datum size, which is opposite to the size of other lines. The grade direction is wrong on the different lines of the same pattern.

Published by Francis Academic Press, UK 

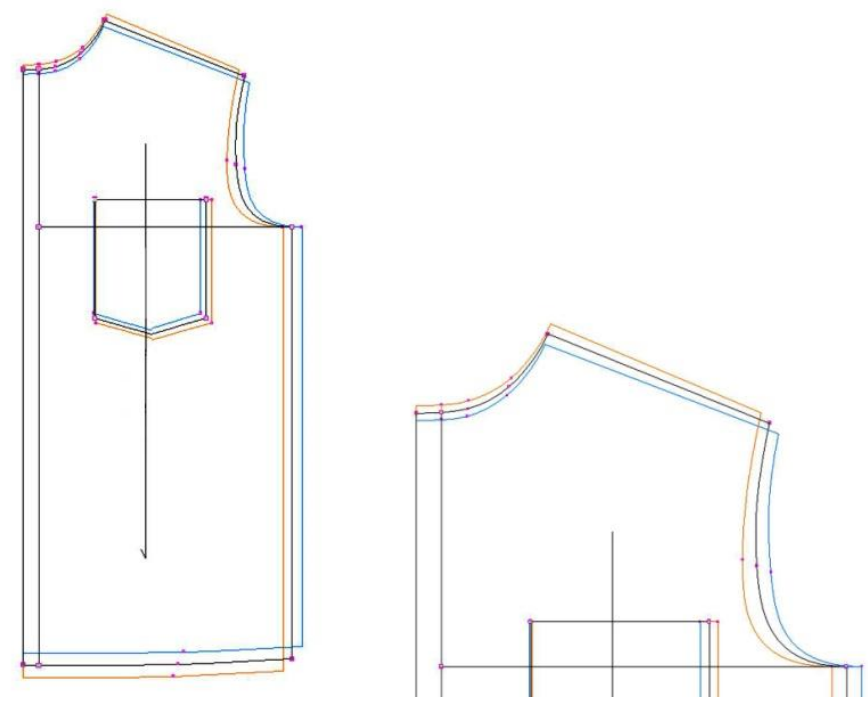

Figure. 2 The pattern of men's shirt

Figure 3 shows the big and small sleeves in the same set of suit pattern. However, the two patterns have conflicting sizes at the elbow and cuff lines of sleeves, that is in the small pattern, the elbow and cuff lines of large size sleeves are below, and the elbow and cuff lines of small size are above. But the big pattern shows the opposite, the elbow and cuff lines of large size are above, and the lines of small size are below. The grade direction is wrong on the same set of pattern.

Published by Francis Academic Press, UK 


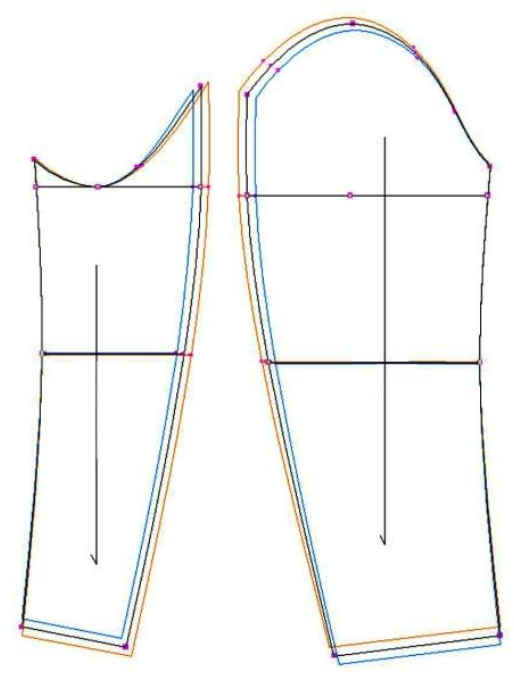

Figure. 3 The pattern of two sleeves

3. Selection of the coordinate axis and calculation of the sign of the pattern grading point

The following will focus on the selection of the vertical and horizontal coordinate axis and the calculation of the sign of the pattern grading point.

\subsection{Selection of the vertical and horizontal coordinate axis}

Pattern grading is the increase or decrease of plane area, so it is necessary to control the growth of two directions in a two-dimensional rectangular coordinate system. The establishment of different rectangular coordinate systems will directly affect the convenience of grading and the judgment of the direction sign of the pattern grading point. The principles of the vertical and horizontal axes are as follows : First, The coordinate axis must be a straight line or an arc with a very small curve rate. Second, for different origin, the grade value of pattern grading point is also different. We should try to select the origin which makes the pattern grading point's grade value to be integral, in order to simplify the calculation and improve the efficiency.

The coordinate axis should be conducive to the proper distance between the contour

Published by Francis Academic Press, UK 
Academic Journal of Computing \& Information Science

ISSN 2616-5775 Vol. 3, Issue 1: 102-113, DOI: 10.25236/AJCIS.2020.030111

lines with large curvature and try to avoid too close to the contour lines of each grade [8]. Based on the above principles, the position relationship between the selected vertical and horizontal coordinate axes and the pattern is summarized, which can be divided into the following categories

\subsubsection{Datum point inside pattern}

In the first type, the intersection of the vertical and horizontal coordinate axes is inside the pattern, as shown in Figure 4, and the pattern is divided into four areas, that is, each pattern grading point in the pattern is located in four quadrants divided by the vertical and horizontal coordinate axes, except for the point coincident with the coordinate. The selection of such coordinate axes is often seen in one sleeve, trousers and overcoats with side and front pattern combined.

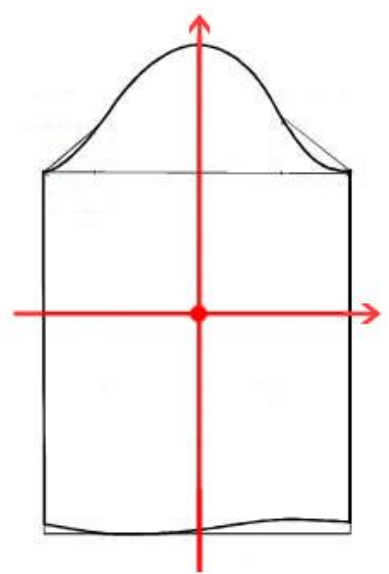

Figure. 4 The first type

\subsubsection{The datum line coincides with one side of the pattern}

The second type is that the vertical or horizontal coordinate axis coincides with one side of the pattern, as shown in Figure 5, that is one side of the pattern is taken as the vertical or horizontal coordinate axis. At this time, each pattern grading point in the pattern is distributed in two quadrants divided by the coordinate axis except for the point coincided with the coordinate. The datum point obtained from the intersection of the vertical and horizontal axes is on one side of the pattern, which becomes one of the pattern grading points. This kind of coordinate axis is often selected in skirts, two sleeves, and most tops.

Published by Francis Academic Press, UK 


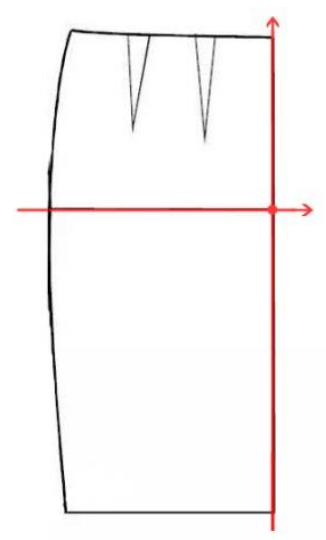

Figure. 5 The second type

\subsubsection{The datum line coincides with both sides of the pattern}

The third type is that the vertical and horizontal coordinate axes coincide with one side of the pattern, as shown in Figure 6, that is one side of the pattern is used as the vertical coordinate axis, and the other side is used as the horizontal coordinate axis. At this time, each pattern grading point in the pattern is distributed in one of the quadrants divided by the coordinate axis except for the point coincided with the coordinate. The datum point obtained from the intersection of the vertical and horizontal coordinate axes is the intersection of the two sides of the coordinate axis in the pattern, and it is also one of the pattern grading points. The selection of such coordinate axes is mostly seen in yoke and other division patterns.

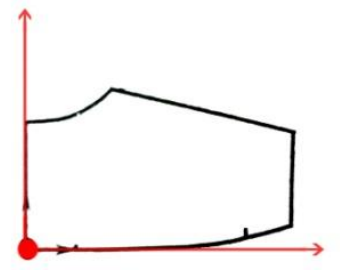

Figure. 6 The third type

\subsubsection{Datum point outside the pattern}

The fourth type is that the intersection point of the vertical and horizontal

Published by Francis Academic Press, UK 
Academic Journal of Computing \& Information Science

ISSN 2616-5775 Vol. 3, Issue 1: 102-113, DOI: 10.25236/AJCIS.2020.030111

coordinate axes is outside the pattern, as shown in Figure 7. Except for the point that coincide with coordinates, the rest points are distributed in two quadrants or one quadrant divided by the coordinate axis, among which one quadrant is rare. The selection of this kind of coordinate axis is mostly seen in the clothing with side piece, that is, the selection of vertical and horizontal coordinate axis of side piece is attached to another pattern. At this time, the datum point resulting from the intersection of the vertical and horizontal axes will be on another pattern, independent of itself.

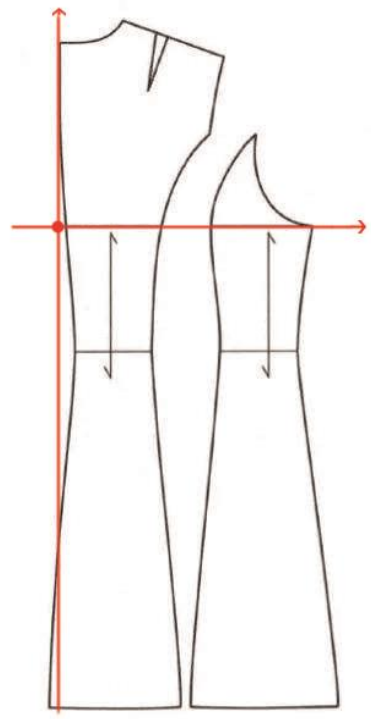

Figure. 7 The fourth type

\subsubsection{Grade in single direction}

In the fifth type, there is only one direction of pattern grading, most of which is only have the horizontal grading value, as shown in Figure 8. In this case, there will be no strict sense of quadrant division and datum point selection. This type is commonly used for pattern grading of collar, cuff, pocket and other parts.

Published by Francis Academic Press, UK 


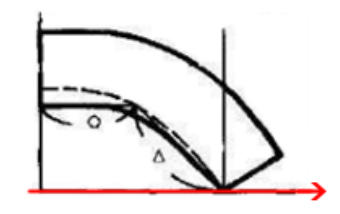

Figure. 8 The fifth type

\subsection{Calculation of the sign of the pattern grading point}

\subsubsection{The pattern grading point is on quadrant}

There are four kinds of situations where the pattern grading point is located in quadrant, namely, the first, the second, the third and the fourth quadrants. The following analysis is the sign judgment relative to the middle size, that is, the reference size : When the pattern grading point is in the first quadrant, the $\mathrm{X}$ and $\mathrm{Y}$ values of the enlarged size are both positive, and the $\mathrm{X}$ and $\mathrm{Y}$ values of the reduced size are both negative ; When the pattern grading point is in the second quadrant, the $\mathrm{X}$ value of the enlarged size is negative, and the $\mathrm{Y}$ value is positive. The sign of the $\mathrm{X}$ and $\mathrm{Y}$ value of the reduced size are opposite to the enlarged size; When the pattern grading point is in the third quadrant, the situation is exactly the opposite of that in the first quadrant, that is, the $\mathrm{X}$ and $\mathrm{Y}$ values of the enlarged size are negative, and the $\mathrm{X}$ and $\mathrm{Y}$ values of the reduced size are positive ; When the pattern grading point is in the fourth quadrant, the situation is exactly the opposite of that in the second quadrant, that is, the $\mathrm{X}$ value of the enlarged size is a positive sign, the $\mathrm{Y}$ value is a negative sign, the $\mathrm{X}$ value of the reduced size is a negative sign, and the $\mathrm{Y}$ value is a positive sign.

\subsubsection{The pattern grading point is on the coordinate axis}

There are also four kinds of situations in which the pattern grading point is located on the coordinate axis, namely, the positive and negative axis of $\mathrm{X}$, the positive and negative axis of $\mathrm{Y}$. Also judge the sign with respect to the middle size: when the pattern grading point is on the $\mathrm{X}$ axis, no matter the positive axis or the negative axis, because the $\mathrm{X}$ axis is the horizontal datum line, the pattern grading point has no pattern grading quantity in the $Y$ direction of the transverse datum line, and its $\mathrm{Y}$ value is 0 . On the positive $\mathrm{X}$ axis, the $\mathrm{X}$ value of the enlarged size is 
Academic Journal of Computing \& Information Science

ISSN 2616-5775 Vol. 3, Issue 1: 102-113, DOI: 10.25236/AJCIS.2020.030111

positive, and the reduced size is negative; on the negative $\mathrm{X}$ axis, it is the opposite. Similarly, when the pattern grading point is on the $\mathrm{Y}$ axis, the $\mathrm{X}$ value is 0 . On the positive $\mathrm{Y}$ axis, the $\mathrm{Y}$ value of the enlarged size is positive, and the reduced size is negative; on the negative $\mathrm{Y}$ axis, it is the opposite.

\subsubsection{The pattern grading point is at the origin}

When the pattern grading point is located at the origin of the coordinate axis, since the origin is the intersection of the longitudinal datum line and the transverse datum line, that is the datum point, and there is no pattern grading quantity in the $\mathrm{X}$ and $\mathrm{Y}$ directions, so the $\mathrm{X}$ and $\mathrm{Y}$ values of the enlarged and reduced sizes are both 0 .

\section{Application of analysis results}

Taking the back center and back side of women's suit as examples, the coordinate axis selection method and direction sign calculation method are applied. The back central line is the longitudinal reference line of the middle back pattern, and the bust line is the transverse reference line. Therefore, the back central line of the pattern coincides with the vertical coordinate, and the whole back center piece is located on one side of the vertical coordinate axis, which is divided into two areas by the horizontal coordinate axis, as shown in Figure 9. Except for the point coincided with the coordinate, the rest points are distributed in the second and third quadrants. Take the grading of the pattern grading point to the enlarged size as an example, analyze the direction sign of each pattern grading point. Point $\mathrm{A}$ is in positive $\mathrm{Y}$ axis, $\mathrm{X}$ value is $0, \mathrm{Y}$ value is positive sign ; Point $\mathrm{B}, \mathrm{C}$ and $\mathrm{D}$ are in the second quadrant, $\mathrm{X}$ is a negative sign, $\mathrm{Y}$ is a positive sign ; Point $\mathrm{F}$ is in the negative $\mathrm{X}$ axis, $\mathrm{X}$ value is a negative sign, $\mathrm{Y}$ value is 0 ; Point $\mathrm{G}$ and $\mathrm{I}$ is in the third quadrant, $\mathrm{X}$ value is negative sign, $\mathrm{Y}$ value is negative sign ; Point $\mathrm{L}$ and $\mathrm{J}$ is in the negative $\mathrm{Y}$ axis, $\mathrm{X}$ value is $0, \mathrm{Y}$ value is negative sign ; Point $\mathrm{E}$ is the intersection of $\mathrm{X}$-axis and $\mathrm{Y}$-axis, so it is the datum point, and both $\mathrm{X}$ and $\mathrm{Y}$ values are 0 . It's just the opposite when grading of the pattern grading point to the reduced size.

The back side of the pattern depends on the selection of the coordinate axis of the back center piece, so the intersection of the vertical and horizontal coordinate axes is outside the back side piece. The coordinate axis divides the pattern into two areas, and the rest points are also distributed in the second and third quadrants except the points coincidence with the coordinate. Similarly, take the grading of the

Published by Francis Academic Press, UK 
Academic Journal of Computing \& Information Science

ISSN 2616-5775 Vol. 3, Issue 1: 102-113, DOI: 10.25236/AJCIS.2020.030111

pattern grading point to the enlarged size as an example to analyze the direction sign of each pattern grading point. Point $D^{\prime}$ is in the second quadrant, $\mathrm{X}$ value is a negative sign, $\mathrm{Y}$ value is a positive sign ; Point $\mathrm{M}$ and $\mathrm{F}^{\prime}$ is in the negative $\mathrm{X}$ axis, $\mathrm{X}$ value is a negative sign, $\mathrm{Y}$ value is 0 ; Point $\mathrm{N}, \mathrm{P}, \mathrm{I}$ ', G' are in the third quadrant, $\mathrm{X}$ value is negative sign, $\mathrm{Y}$ value is negative sign. It's just the opposite when grading of the pattern grading point to the reduced size.

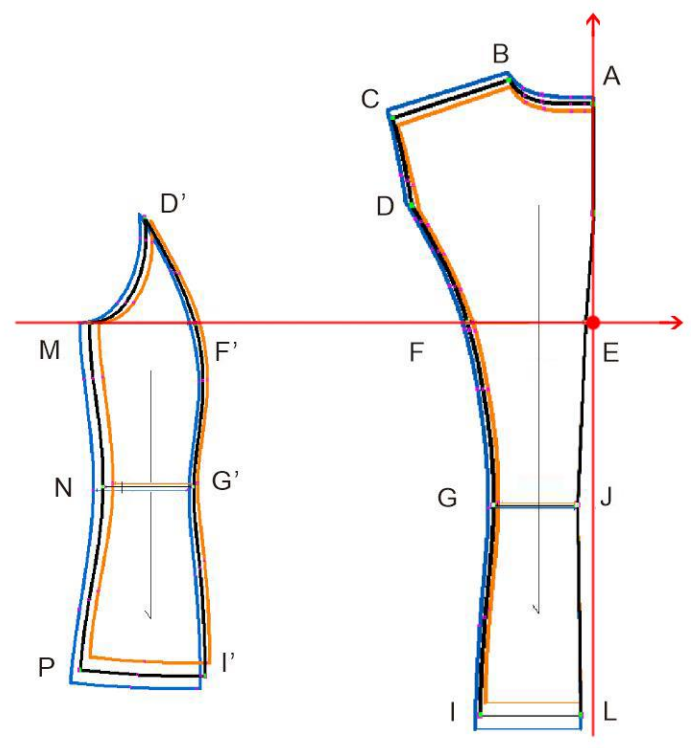

Figure. 9 The back center and back side of women's suit

\section{Conclusin}

There are many ways to grade, but either way needs to establish a rectangular coordinate system. Starting from the basic principle, truly understanding and mastering the selection method of coordinate axis and the judgment principle of coordinate point direction will be the key to solve the confusion of value input of pattern grading CAD system and the fundamental method to improve the operation efficiency of operators when using the pattern grading CAD system.

Published by Francis Academic Press, UK 
Academic Journal of Computing \& Information Science

ISSN 2616-5775 Vol. 3, Issue 1: 102-113, DOI: 10.25236/AJCIS.2020.030111

\section{Acknowledgements}

Project fund: 2019 domestic visiting engineer of institutions of higher learning "school-enterprise cooperation project" (FG2019052) ; 2018-2019 school year of Wenzhou Polytechnic "13th five-year plan" education and teaching reform project (WZYzd201904).

\section{References}

[1] Ziying Yu, Luyi Chen and $\mathrm{Xu}$ Li (2005). Technology development and evaluation system of clothing CAD. Journal of Zhejiang Sci-Tech University, vol.22, no.4, p.350-355.

[2] Lanyou Li et al.(1997) Principle and application of clothing CAD. Beijing: China Textile Press,

[3] Qi Zhou (2003). Development and research on the continuous garment CAD platform-Men suit collection. MA.Sc: Tianjin Polytechnic University.

[4] Zhen Han(2008). Research on Automatic Pattern Grading Techniques in Compter Aided Garment Design. MA.Sc: Beijing Institute Of Fashion Technology.

[5] Wenjie Ding, Yi Cao (2008). Study on dress lofting techniques based on AutoCAD2000. Journal of Southern Yangtze University (Natural Science Edition), vol.1, no.4, p.341-344.

[6] Huaxia Zhang(2015). A comparative analysis of the method of pattern grading in clothing CAD software. Tianjin Textile Science \& Technology, no.4, p.32-33.

[7] Xiaolu Ye(2018). Deal with the direction of pattern grading point problems in computer aided garment design point grading method. Progress in Textile Science \& Technology,no.1,p.46-49.

[8] Fenyuan Zou(2012). The principls and techniques of clothing industrial pattern making. Hangzhou: Zhejiang University Press,

Published by Francis Academic Press, UK 